\title{
Undiagnosed diabetes, hypertension, and hypercholesterolaemia in an overweight or obese population: implications for cardiovascular disease risk screening programme
}

\author{
Upul Senarath ${ }^{1}$, Prasad Katulanda ${ }^{2}$, Dulitha Fernando ${ }^{1}$, Nishan Sudheera Kalupahana ${ }^{3}$, \\ Kunarathinam Partheepan ${ }^{1}$, Gaya Katulanda ${ }^{4}$, Michael Dibley ${ }^{5}$ \\ (Index words: undiagnosed diabetes, undiagnosed hypertension, undiagnosed hypercholesterolaemia, cardiovascular \\ disease risk, screening)
}

\begin{abstract}
Introduction Establishing the burden of undiagnosed CVD risk factors is critical to monitoring public health efforts related to screening and diagnosis.

Objective To assess the proportion and determinants of undiagnosed diabetes, hypertension, and hypercholesterolaemia, among overweight or obese adults.

Methods A sample of 1200 participants aged 35-64 years with a BMI $\geq 25 \mathrm{~kg} / \mathrm{m}^{2}$ was selected from the Colombo district. Data were collected through a questionnaire, anthropometry, blood pressure measurement, and blood sampling for fasting plasma glucose, $\mathrm{HbA1c}$, and lipid profile. Undiagnosed diabetes, hypertension, and hypercholesterolaemia were defined as fasting plasma glucose $\geq 126 \mathrm{mg} / \mathrm{dL}$ or $\mathrm{HbA} 1 \mathrm{c} \geq 6.5 \%$; systolic blood pressure $\geq 140 \mathrm{mmHg}$ or diastolic blood pressure $\geq 90 \mathrm{mmHg}$; total cholesterol $\geq 240 \mathrm{mg} / \mathrm{dl}$ respectively, in a person without a previous diagnosis. Multiple logistic regression analyses were carried out to identify determinants.

Results The prevalence $(95 \% \mathrm{Cl})$ of diabetes was $28.0 \%$ $(25.5,30.5)$, hypertension, 33.4\% $(30.7,36.1)$ and hypercholesterolaemia, $31.9 \%(29.2,34.5)$. The proportion of undiagnosed diabetes was $13.8 \%$ (11.9, 15.8), undiagnosed hypertension $11.3 \%(9.5,13.1)$, and undiagnosed hypercholesterolaemia $17.8 \%(15.6,19.9)$. Undiagnosed cases accounted for almost half of all diabetes cases, one-third of all hypertension cases, and more than half $(56 \%)$ of all high cholesterol cases. The key determinants for undiagnosed CVD risk were: male sex, low or middle income, rural residence, and relatively younger age.

Conclusion CVD screening programmes should be tailored to target populations based on these determinants and provide basic diagnostic facilities in all
\end{abstract}

health centres. The 'proportion undiagnosed' in the population may be a useful indicator to evaluate their effectiveness.

Ceylon Medical Journal 2020; 65: 46-55

DOI: http://doi.org/10.4038/cmj.v65i3.9185

\section{Introduction}

Cardiovascular diseases (CVD) are the leading cause of death globally. In 2015, there were an estimated 422.7 million cases and 17.9 million deaths due to CVD in the world [1]. According to the Global Burden of Disease 2016 Study, CVDs accounted for $20 \%$ of the total disease burden in women and $24 \%$ of the total burden in men [2]. Ischaemic heart disease, causing 174 million Disability Adjusted Life Years (DALY), remained the leading cause of total global CVD burden. In Sri Lanka, ischaemic heart disease is the leading cause of death and was responsible for 31.0 deaths per 100,000 population in 2017 [3]. If the health systems fail to respond to this burden using a scientific approach, the Sustainable Development Goal (SDG) target of reducing premature deaths due to noncommunicable diseases by one-third by 2030 will be far from reality [2,4].

There are multiple risk factors that contribute to cardiovascular risk. Abnormal lipids, smoking, hypertension, diabetes, abdominal obesity, psychosocial factors, low consumption of fruits and vegetables, high alcohol consumption, and physical inactivity account for most of the CVD risk worldwide [5]. The WHO emphasizes that people who are at high cardiovascular risk due to the

${ }^{1}$ Department of Community Medicine, Faculty of Medicine, University of Colombo, Sri Lanka, ${ }^{2}$ Department of Clinical Medicine, Faculty of Medicine, University of Colombo, Sri Lanka, ${ }^{3}$ Department of Physiology, Faculty of Medicine, University of Peradeniya, Sri Lanka, ${ }^{4}$ Department of Pathology, National Hospital of Sri Lanka, ${ }^{5}$ The Sydney School of Public Health, Faculty of Medicine and Health, The University of Sydney, NSW, Australia.

Correspondence: US, e-mail: <upul@commed.cmb.ac.lk>. Received 20 May 2020 and revised version 30 July 2020 accepted 20 October 2020.

This is an open-access article distributed under the terms of the Creative Commons Attribution License, which permits unrestricted use, distribution, and reproduction in any medium, provided the original author and source are credited. 
presence of one or more risk factors such as hypertension, diabetes, hyperlipidaemia or already established disease, need early detection and management using counselling and medicines, as appropriate [6].

In Sri Lanka, Healthy Lifestyle Centers (HLC) were established by the Ministry of Health in 2011 as a noncommunicable disease (NCD) screening service at primary health care institutions. By the first quarter of 2016, the cumulative percentage of the target population (aged 40-65 years) screened for NCD risk factors at HLC was $25.5 \%$, with a lower proportion of men than women [7]. This percentage is still low in contrast to the rising trend of CVD, and recent improvement in availability and readiness of services at the HLC $[3,8,9]$. With this low coverage, there is a high chance that many individuals in the population are living with CVD risk factors such as diabetes, hypertension, and hypercholesterolaemia without knowing their presence. Thus, establishing the burden of undiagnosed CVD risk factors is critical to monitoring public health efforts related to screening and diagnosis [10].

It has been previously described by the Sri Lanka Diabetes Cardiovascular Study (SLDCS 2005-06) that the prevalence of undiagnosed diabetes was 3.9\% in the adult population. The undiagnosed diabetes accounted for onethird of all diabetes cases [11]. There are no recently published data regarding the proportion of undiagnosed as a percentage of the population. Furthermore, any previous in-country studies for undiagnosed hypertension or hypercholesterolaemia are not available. Little is known about the type of people who are more likely to be undiagnosed for CVD risk factors. Potential determinants for undiagnosed CVD risk factors would be useful in identifying target populations for efficient screening. In this study, we aimed to assess the proportion and determinants of undiagnosed diabetes, hypertension, and hypercholesterolaemia, among overweight or obese adults aged 35-64 years from the Colombo district in Sri Lanka.

\section{Methods}

\section{Study population}

The present study included cross-sectional data of the baseline assessment of a randomized controlled trial designed to evaluate the effects of a mHealth nutrition and lifestyle intervention on CVD risk reduction in the Colombo district. Individuals aged 35-64 years with a body mass index (BMI) of $25 \mathrm{~kg} / \mathrm{m}^{2}$ or higher were included. Currently, pregnant or breastfeeding mothers and anyone who has a recent weight loss of at least $5 \%$ in the preceding six months was excluded. The estimated sample size was 1200 to test a reduction in 10-year CVD risk among participants by one-third, following the intervention.

Participants were selected in two stages from the 15 $\mathrm{MOH}$ areas in the Colombo Regional Director of Health
Services area. In the first stage, 5 to 6 clusters (Grama Niladhari Divisions) within each $\mathrm{MOH}$ area were selected randomly. In the second stage, data collectors visited about 30-35 households located together within each selected cluster. The potential participants were screened for eligibility (as described above), and BMI was calculated by measuring weights and heights during this process. Only one eligible participant was chosen randomly from a given family. Of the 2518 participants screened for eligibility, 1318 were excluded due to noneligibility. A detailed methodology is available elsewhere [12].

The project obtained ethics clearance from the Ethics Review Committee of the Faculty of Medicine, University of Colombo (Ref. No: EC-16-061).

\section{Measurements}

Basic sociodemographic data (age, sex, ethnicity, education level, employment, income, and residence) were gathered through a questionnaire-based interview. Selfreported physician diagnoses of diabetes, hypertension, and hypercholesterolaemia were verified through medical records or prescriptions. Height and weight were measured using standard anthropometric equipment to the nearest $0.1 \mathrm{~cm}$ and $0.1 \mathrm{~kg}$, respectively (Seca 213 and 813, Hamburg, Germany). Blood pressure was measured three times at the right arm in sitting position after a fifteen-minute rest using a validated digital blood pressure apparatus (Omron HEM 7320, Kyoto, Japan). The average of the second and third measurements was used. Blood samples $(6 \mathrm{ml}$ of blood per person) were drawn in 12-hour fasting state and sent to an accredited laboratory within an hour and tested using the recommended methods - fasting plasma glucose using glucose oxidase method (Randox Imola, UK), HbA1c using high-performance liquid chromatographic assay (Bio-Rad D10, USA), and total cholesterol using cholesterol oxidase method (Randox Imola, UK).

\section{Definitions of diagnosed and undiagnosed risk factors}

Undiagnosed diabetes was defined as elevated levels of either fasting glucose ( $\geq 7.0 \mathrm{mmol} / \mathrm{L}$ [ $\geq 126 \mathrm{mg} / \mathrm{dL}])$ or HbA1c ( $\geq 6.5 \%)$ measured in the same blood sample in a person without a previous diagnosis of diabetes. Diagnosed diabetes was defined as a self-reported physician diagnosis of diabetes or receiving medication for diabetes. Persons defined as not having diabetes included those with fasting glucose $<126 \mathrm{mg} / \mathrm{dL}$ and HbA1c $<6.5 \%$. The classification was based on the American Diabetes Association (ADA) diagnostic criteria [13].

Undiagnosed hypertension was defined as elevated levels of systolic blood pressure ( $\geq 140 \mathrm{mmHg}$ ) or diastolic blood pressure ( $\geq 90 \mathrm{mmHg}$ ), measured in a person without a previous diagnosis of hypertension. Diagnosed hypertension was defined as a self-reported physician diagnosis of raised blood pressure or current use of 
antihypertensive medication. Persons defined as not having hypertension included those with systolic and diastolic blood pressure $<140 \mathrm{mmHg}$ and $<90 \mathrm{mmHg}$ respectively.

Undiagnosed hypercholesterolaemia was defined as elevated levels of total cholesterol ( $\geq 240 \mathrm{mg} / \mathrm{dl}$ ) measured in a person without a previous diagnosis of hypercholesterolaemia. Diagnosed hypercholesterolaemia was defined as a self-reported physician diagnosis of raised cholesterol or receiving medication for it. Persons defined as not having hypercholesterolaemia included those with normal total cholesterol ( $<240 \mathrm{mg} / \mathrm{dl})$.

For self-reported physician diagnosis of any of the conditions, verification was done by review of previous medical records, laboratory reports, or prescriptions.

\section{Statistical analyses}

Percentages with 95\% confidence intervals were calculated for undiagnosed, diagnosed, and not having disease out of the total investigated. The undiagnosed CVD risk conditions (diabetes, hypertension or hypercholesterolemia) were expressed as a percentage of all participants. It reflects the probability that a person in the defined population to have the undiagnosed risk condition. Undiagnosed percentages were disaggregated by biological and sociodemographic variables.

Multiple logistic regression analyses were carried out using undiagnosed as the dependent variable (undiagnosed $=1$; previously diagnosed $=0$ ) while entering all biological and socioeconomic variables as categorical covariates in a single step. The purpose of the regression analysis is to estimate the magnitude of effect and significance of independent variables for predicting the 'undiagnosed' in contrast to the 'previously diagnosed' after adjusting for confounding variables. Thus, the category of 'not having disease' was excluded in the regression models. Adjusted odds ratios (95\% confidential interval) are presented to indicate the magnitude of risk (odds of being undiagnosed) with $\mathrm{p}<0.05$ as the level of significance. SPSS version 20.0 was used for statistical analysis.

\section{Results}

Sociodemographic characteristics of participants

Table 1 presents the distribution of participants according to selected biological and sociodemographic characteristics. Of the 1200 participants, 64\% were female, $60 \%$ aged below 50 years, and $70 \%$ were overweight with BMI $25-29.9 \mathrm{~kg} / \mathrm{m}^{2}$. The proportions of Sinhalese, Tamil, and Moor ethnicities were 95.5\%, 2.1\%, and 2.2\% respectively. More than two-thirds (67.4\%) had completed GCE (O/L) or above. Approximately $27 \%$ were not engaged in any employment, and $12 \%$ reported a monthly household income of less than 20,000 LKR. Seventy percent live in urban areas.

Table 1. Biological and sociodemographic characteristics of participants $(n=1200)$

\begin{tabular}{llrr}
\hline Characteristic & & No. & $\%$ \\
\hline \multirow{2}{*}{ Sex } & Male & & $36.2 \%$ \\
& Female & 435 & $63.8 \%$ \\
Age category (years) & $35-39$ & 765 & $16.3 \%$ \\
& $40-44$ & 196 & $22.0 \%$ \\
& $45-49$ & 264 & $21.2 \%$ \\
& $50-54$ & 255 & $19.8 \%$ \\
BMI category $\left(\mathrm{kg} / \mathrm{m}^{2}\right)$ & $55-59$ & 238 & $13.1 \%$ \\
& $60-64$ & 157 & $7.5 \%$ \\
& $25.00-27.49$ & 90 & $40.7 \%$ \\
& $27.50-29.99$ & 488 & $28.8 \%$ \\
& $30.00-32.49$ & 345 & $16.1 \%$ \\
& $32.50-34.99$ & 193 & $7.5 \%$ \\
& $35.00-39.99$ & 90 & $5.7 \%$ \\
& $40.00 \&$ above & 68 & $1.3 \%$ \\
& Sinhalese & 16 & $95.5 \%$ \\
& Tamil & 1146 & $2.1 \%$ \\
& Moor & 25 & $2.2 \%$ \\
& Other & 27 & $0.2 \%$ \\
\hline
\end{tabular}




\begin{tabular}{|c|c|c|c|}
\hline Characteristic & & No. & $\%$ \\
\hline \multirow[t]{5}{*}{ Highest level of education } & None or Primary (Grades 1-5) & 54 & $4.5 \%$ \\
\hline & Secondary (Grades 6-11) & 338 & $28.2 \%$ \\
\hline & GCE OL passed & 272 & $22.7 \%$ \\
\hline & GCE AL passed & 354 & $29.5 \%$ \\
\hline & Degree and above & 182 & $15.2 \%$ \\
\hline \multirow[t]{7}{*}{ Nature of employment } & Full-time public sector & 491 & $40.9 \%$ \\
\hline & Full-time private sector & 132 & $11.0 \%$ \\
\hline & Self-employed/own account employer & 176 & $14.7 \%$ \\
\hline & Casual employment & 14 & $1.2 \%$ \\
\hline & Unpaid family work & 42 & $3.5 \%$ \\
\hline & Retired & 18 & $1.5 \%$ \\
\hline & Unemployed & 327 & $27.2 \%$ \\
\hline \multirow[t]{5}{*}{ Monthly income (LKR) } & $<20,000$ & 142 & $11.8 \%$ \\
\hline & 20,000-39,999 & 495 & $41.2 \%$ \\
\hline & $40,000-59,999$ & 357 & $29.8 \%$ \\
\hline & $60,000-79,999$ & 111 & $9.2 \%$ \\
\hline & $80,000 \&$ above & 95 & $7.9 \%$ \\
\hline \multirow[t]{2}{*}{ Residential sector } & Rural & 360 & $30.0 \%$ \\
\hline & Urban & 840 & $70.0 \%$ \\
\hline
\end{tabular}

Diabetes, hypertension, and hypercholesterolaemia

In this subpopulation, the prevalence $(95 \% \mathrm{CI})$ of diabetes was $28.0 \%$ (25.5, 30.5), hypertension, 33.4\% (30.7, 36.1) and hypercholesterolaemia, 31.9\% (29.2, 34.5).

As illustrated in Figure 1, the proportion (95\%CI) undiagnosed diabetes was 13.8\% (11.9,15.8), undiagnosed hypertension $11.3 \%(9.5,13.1)$, and undiagnosed hyper- cholesterolaemia $17.8 \%(15.6,19.9)$. The proportion $(95 \%$ CI) of previously diagnosed diabetes was $14.2 \%$ (12.2, $16.1)$, hypertension $22.1 \%(19.7,24.4)$ and hypercholesterolaemia $14.1 \%$ (12.1, 16.1). Undiagnosed cases accounted for almost half of all diabetes cases, one-third of all hypertension cases, and more than half (56\%) of all high cholesterol cases.

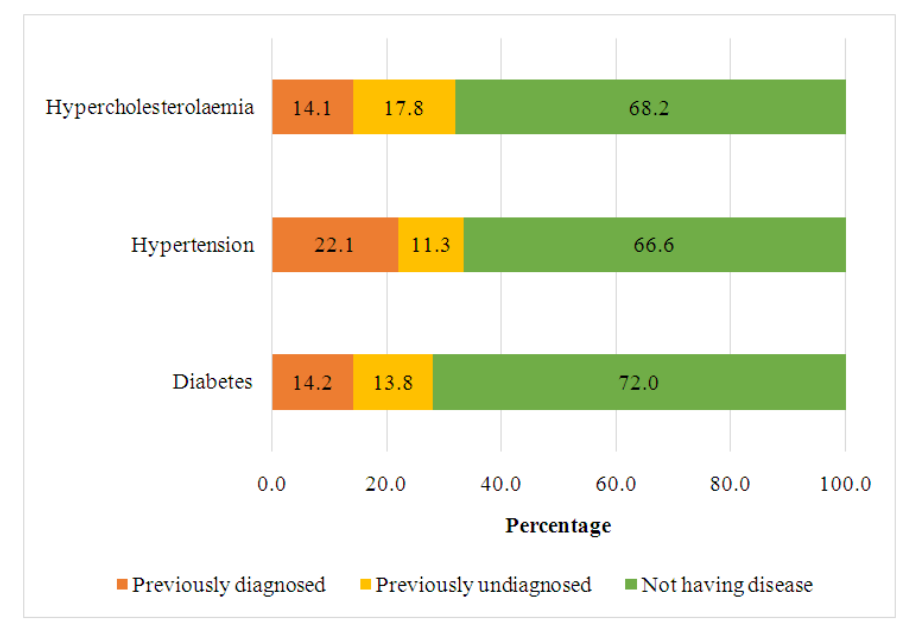

Figure 1. Diagnosed and undiagnosed diabetes, hypertension and hypercholesterolaemia ( $n=1200)$.

Percentages and 95\% CI:

Previously diagnosed: diabetes $14.2 \%$ (12.2, 16.1); hypertension $22.1 \%(19.7,24.4)$;

hypercholesterolaemia $14.1 \%(12.1,16.1)$.

Previously undiagnosed: diabetes $13.8 \%(11.9,15.8)$;

hypertension $11.3 \%$ (9.5, 13.1); hypercholesterolaemia 17.8\% (15.6, 19.9). 
Determinants of undiagnosed risk factors

The results of multiple logistic regression analyses are presented in Tables 2 to 4 . The odds ratios indicate the odds of being undiagnosed for a given predictor variable when adjusted for all other variables in the table.

People living in rural areas $(\mathrm{AOR}=2.058)$, having income less than LKR 60,000 per month (AOR ranged from 2.671 to 2.813 for different income groups), or who were marginally obese $\left(\mathrm{AOR}=3.056\right.$ for BMI $30.00-32.5 \mathrm{~kg} / \mathrm{m}^{2}$ ) were more likely to be undiagnosed with diabetes (Table 2). Men were almost twice ( $\mathrm{AOR}=2.034)$ as likely to be undiagnosed with hypertension than women. There was no urban/rural difference in undiagnosed hypertension.

Table 2. Determinants of undiagnosed diabetes: multiple logistic regression analysis

\begin{tabular}{|c|c|c|c|c|c|c|}
\hline \multirow{2}{*}{$\begin{array}{l}\text { Characteristic } \\
\text { Sex }\end{array}$} & \multirow[b]{2}{*}{ Male } & \multirow{2}{*}{$\begin{array}{c}\begin{array}{c}\text { Undiagnosed } \\
\text { diabetes }\end{array} \% \\
13.8 \%\end{array}$} & \multirow{2}{*}{$\begin{array}{l}P \text { value }^{\mathrm{b}} \\
0.085\end{array}$} & \multirow{2}{*}{$\begin{array}{c}\text { Adjusted } \\
\text { OR } \\
0.624\end{array}$} & \multicolumn{2}{|c|}{$\begin{array}{l}\text { 95\% Confidence } \\
\text { Interval }\end{array}$} \\
\hline & & & & & 0.364 & 1.067 \\
\hline & Female & $13.9 \%$ & - & - & - & - \\
\hline \multirow[t]{6}{*}{ Age category (years) } & $60-64$ & $20.0 \%$ & 0.180 & 0.490 & 0.172 & 1.391 \\
\hline & $55-59$ & $16.6 \%$ & 0.022 & 0.332 & 0.129 & .854 \\
\hline & $50-54$ & $16.0 \%$ & 0.024 & 0.356 & 0.145 & .873 \\
\hline & $45-49$ & $14.9 \%$ & 0.078 & 0.443 & 0.180 & 1.095 \\
\hline & $40-44$ & $10.2 \%$ & 0.809 & 0.882 & 0.319 & 2.436 \\
\hline & $35-39$ & $9.7 \%$ & - & - & - & - \\
\hline \multirow[t]{6}{*}{ BMI category $\left(\mathrm{kg} / \mathrm{m}^{2}\right)$} & $40.00 \&$ above & $25.0 \%$ & 0.125 & 5.831 & 0.611 & 55.614 \\
\hline & $35.00-39.99$ & $17.6 \%$ & 0.365 & 1.545 & 0.603 & 3.962 \\
\hline & $32.50-34.99$ & $16.7 \%$ & 0.090 & 2.212 & 0.883 & 5.537 \\
\hline & $30.00-32.49$ & $16.6 \%$ & 0.002 & 3.056 & 1.523 & 6.135 \\
\hline & 27.50-29.99 & $15.1 \%$ & 0.132 & 1.518 & 0.881 & 2.614 \\
\hline & $25.00-27.49$ & $10.5 \%$ & - & - & - & - \\
\hline \multirow[t]{3}{*}{ Ethnicity } & Moor & $33.3 \%$ & 0.314 & 1.837 & 0.563 & 5.993 \\
\hline & Tamil & $16.0 \%$ & 0.699 & 1.323 & 0.319 & 5.489 \\
\hline & Sinhalese & $13.4 \%$ & - & - & - & - \\
\hline \multirow[t]{5}{*}{ Highest level of education } & None or Primary & $22.2 \%$ & 0.370 & 0.558 & 0.156 & 1.997 \\
\hline & Secondary & $17.2 \%$ & 0.464 & 0.688 & 0.253 & 1.870 \\
\hline & GCE OL passed & $12.5 \%$ & 0.191 & 0.519 & 0.194 & 1.387 \\
\hline & GCE AL passed & $11.6 \%$ & 0.394 & 0.682 & 0.283 & 1.644 \\
\hline & Degree and above & $11.5 \%$ & - & - & - & - \\
\hline \multirow[t]{7}{*}{ Nature of employment } & Full-time public sector & $13.4 \%$ & 0.095 & 1.908 & 0.893 & 4.076 \\
\hline & Full-time private sector & $13.6 \%$ & 0.365 & 1.489 & 0.630 & 3.523 \\
\hline & $\begin{array}{l}\text { Self-employed / own } \\
\text { account employer }\end{array}$ & $15.9 \%$ & 0.929 & 0.969 & 0.479 & 1.957 \\
\hline & Casual employment & $7.1 \%$ & 0.750 & 0.662 & 0.052 & 8.367 \\
\hline & Unpaid family work & $14.3 \%$ & 0.671 & 1.324 & 0.362 & 4.838 \\
\hline & Retired & $22.2 \%$ & 0.107 & 4.548 & 0.720 & 28.720 \\
\hline & Unemployed & $13.1 \%$ & - & - & - & - \\
\hline \multirow[t]{5}{*}{ Monthly income (LKR) } & $<20,000$ & $19.0 \%$ & 0.091 & 2.712 & 0.853 & 8.624 \\
\hline & 20,000-39,999 & $14.3 \%$ & 0.060 & 2.671 & 0.960 & 7.436 \\
\hline & $40,000-59,999$ & $15.1 \%$ & 0.042 & 2.813 & 1.037 & 7.628 \\
\hline & $60,000-79,999$ & $3.6 \%$ & 00.238 & 0.434 & 0.109 & 1.734 \\
\hline & $80,000 \&$ above & $10.5 \%$ & - & & & \\
\hline \multirow[t]{2}{*}{ Residential sector } & Rural & $16.1 \%$ & 0.005 & 2.058 & 1.237 & 3.423 \\
\hline & Urban & $12.9 \%$ & - & - & - & - \\
\hline
\end{tabular}

a As a proportion of total investigated

${ }^{\mathrm{b}} \mathrm{P}$ values are for the adjusted odds ratios based on the multiple logistic regression analyses. The dependent variable was defined as 1 if undiagnosed diabetes and 0 if previously diagnosed diabetes. The odds ratio for a given variable was adjusted for all other co-variables in the table. 
Low-income categories did not show significant odds, although the values were somewhat higher for such groups (Table 3). Low and middle-income categories were more likely to be undiagnosed with hypercholesterolaemia (AOR ranged from 3.121 to 3.922 for different income groups) in contrast to the highest monthly income group (Table 4). The results also indicated that older groups were less likely to be undiagnosed with diabetes and hypercholesterolaemia, independent of other sociodemographic factors.

Table 3. Determinants of undiagnosed hypertension: multiple logistic regression analysis

\begin{tabular}{|c|c|c|c|c|c|c|}
\hline \multirow{2}{*}{$\begin{array}{l}\text { Characteristic } \\
\text { Sex }\end{array}$} & \multirow[b]{2}{*}{ Male } & \multirow{2}{*}{$\begin{array}{c}\begin{array}{c}\text { Undiagnosed } \\
\text { diabetes }\end{array} \% \\
16.1 \%\end{array}$} & \multirow{2}{*}{$\begin{array}{l}\text { P value } \\
0.007\end{array}$} & \multirow{2}{*}{$\begin{array}{c}\text { Adjusted } \\
\text { OR } \\
2.034\end{array}$} & \multicolumn{2}{|c|}{$\begin{array}{l}\text { 95\% Confidence } \\
\text { Interval }\end{array}$} \\
\hline & & & & & 1.216 & 3.401 \\
\hline & Female & $8.6 \%$ & - & - & - & - \\
\hline \multirow[t]{6}{*}{ Age category (years) } & $60-64$ & $10.0 \%$ & 0.117 & 0.444 & 0.161 & 1.227 \\
\hline & $55-59$ & $19.7 \%$ & 0.708 & 0.858 & 0.387 & 1.906 \\
\hline & $50-54$ & $10.1 \%$ & 0.133 & 0.539 & 0.240 & 1.208 \\
\hline & $45-49$ & $12.2 \%$ & 0.998 & 1.001 & 0.451 & 2.222 \\
\hline & $40-44$ & $9.5 \%$ & 0.702 & 1.178 & 0.510 & 2.722 \\
\hline & $35-39$ & $8.2 \%$ & - & - & - & - \\
\hline \multirow[t]{6}{*}{ BMI category $\left(\mathrm{kg} / \mathrm{m}^{2}\right)$} & $40.00 \&$ above & $31.2 \%$ & 0.460 & 1.663 & 0.431 & 6.415 \\
\hline & 35.00-39.99 & $16.2 \%$ & 0.886 & 1.064 & 0.454 & 2.492 \\
\hline & $32.50-34.99$ & $15.6 \%$ & 0.153 & 1.812 & 0.801 & 4.099 \\
\hline & $30.00-32.49$ & $8.8 \%$ & 0.176 & 0.630 & 0.322 & 1.231 \\
\hline & 27.50-29.99 & $10.4 \%$ & 0.303 & 0.752 & 0.438 & 1.293 \\
\hline & $25.00-27.49$ & $10.9 \%$ & - & - & - & - \\
\hline \multirow[t]{3}{*}{ Ethnicity } & Moor & $11.1 \%$ & 0.598 & 0.684 & 0.167 & 2.807 \\
\hline & Tamil & $4.0 \%$ & 0.226 & 0.267 & 0.031 & 2.268 \\
\hline & Sinhalese & $11.4 \%$ & - & - & - & - \\
\hline \multirow[t]{5}{*}{ Highest level of education } & None or Primary & $11.1 \%$ & 0.367 & 0.552 & 0.152 & 2.008 \\
\hline & Secondary & $10.9 \%$ & 0.216 & 0.569 & 0.233 & 1.389 \\
\hline & GCE OL passed & $11.8 \%$ & 0.321 & 0.647 & 0.274 & 1.529 \\
\hline & GCE AL passed & $10.5 \%$ & 0.114 & 0.544 & 0.255 & 1.159 \\
\hline & Degree and above & $13.2 \%$ & - & - & - & - \\
\hline \multirow[t]{7}{*}{ Nature of employment } & Full-time public sector & $13.8 \%$ & 0.136 & 1.757 & 0.837 & 3.690 \\
\hline & Full-time private sector & $10.6 \%$ & 0.478 & 0.734 & 0.312 & 1.726 \\
\hline & $\begin{array}{l}\text { Self-employed / own } \\
\text { account employer }\end{array}$ & $13.1 \%$ & 0.149 & 1.717 & 0.824 & 3.578 \\
\hline & Casual employment & $7.1 \%$ & 0.859 & 1.256 & 0.101 & 15.686 \\
\hline & Unpaid family work & $4.8 \%$ & 0.751 & 0.767 & 0.150 & 3.928 \\
\hline & Rtired & $16.7 \%$ & 0.738 & 0.741 & 0.128 & 4.305 \\
\hline & Unemployed & $7.6 \%$ & - & - & - & - \\
\hline \multirow[t]{5}{*}{ Monthly income (LKR) } & $<20,000$ & $10.6 \%$ & 0.262 & 1.890 & 0.621 & 5.745 \\
\hline & 20,000-39,999 & $12.1 \%$ & 0.086 & 2.256 & 0.891 & 5.713 \\
\hline & $40,000-59,999$ & $12.0 \%$ & 0.492 & 1.371 & 0.558 & 3.372 \\
\hline & $60,000-79,999$ & $6.3 \%$ & 0.416 & 0.626 & 0.202 & 1.939 \\
\hline & $80,000 \&$ above & $11.6 \%$ & - & - & - & - \\
\hline \multirow[t]{2}{*}{ Residential sector } & Rural & $9.2 \%$ & 0.833 & 0.947 & 0.572 & 1.568 \\
\hline & Urban & $12.3 \%$ & - & - & - & - \\
\hline
\end{tabular}

${ }^{a}$ As a proportion of total investigated

${ }^{\mathrm{b}} \mathrm{P}$ values are for the adjusted odds ratios based on the multiple logistic regression analyses. The dependent variable was defined as 1 if undiagnosed hypertension and 0 if previously diagnosed hypertension. The odds ratio for a given variable was adjusted for all other co-variables in the table. 
Table 4. Determinants of undiagnosed hypercholesterolemia: multiple logistic regression analysis

\begin{tabular}{|c|c|c|c|c|c|c|}
\hline \multirow{2}{*}{$\begin{array}{l}\text { Characteristic } \\
\text { Sex }\end{array}$} & \multirow[b]{2}{*}{ Male } & \multirow{2}{*}{$\begin{array}{c}\begin{array}{c}\text { Undiagnosed } \\
\text { hypercho- } \\
\text { lesterolemia }\end{array} \% \\
17.0 \%\end{array}$} & \multirow{2}{*}{$\begin{array}{l}\text { P value }{ }^{\mathrm{b}} \\
0.418\end{array}$} & \multirow{2}{*}{$\begin{array}{c}\text { Adjusted } \\
\text { OR } \\
.810\end{array}$} & \multicolumn{2}{|c|}{$\begin{array}{l}\text { 95\% Confidence } \\
\text { Interval }\end{array}$} \\
\hline & & & & & 0.488 & 1.347 \\
\hline & Female & $18.2 \%$ & - & - & - & - \\
\hline \multirow[t]{6}{*}{ Age category (years) } & $60-64$ & $23.3 \%$ & 0.099 & 0.464 & 0.186 & 1.155 \\
\hline & $55-59$ & $14.0 \%$ & 0.010 & 0.331 & 0.142 & 0.771 \\
\hline & $50-54$ & $22.3 \%$ & 0.142 & 0.573 & 0.273 & 1.206 \\
\hline & $45-49$ & $16.9 \%$ & 0.167 & 0.580 & 0.268 & 1.255 \\
\hline & $40-44$ & $15.2 \%$ & 0.130 & 0.548 & .0252 & 1.194 \\
\hline & $35-39$ & $17.3 \%$ & - & - & - & - \\
\hline \multirow[t]{6}{*}{ BMI category $\left(\mathrm{kg} / \mathrm{m}^{2}\right)$} & $40.00 \&$ above & $6.2 \%$ & 0.822 & 0.724 & 0.044 & 12.015 \\
\hline & 35.00-39.99 & $8.8 \%$ & 0.049 & 0.333 & 0.112 & 0.993 \\
\hline & $32.50-34.99$ & $11.1 \%$ & 0.475 & 0.700 & 0.264 & 1.859 \\
\hline & $30.00-32.49$ & $18.7 \%$ & 0.697 & 0.887 & 0.485 & 1.622 \\
\hline & 27.50-29.99 & $16.5 \%$ & 0.210 & 0.728 & 0.443 & 1.196 \\
\hline & $25.00-27.49$ & $21.1 \%$ & - & - & - & - \\
\hline \multirow[t]{3}{*}{ Ethnicity } & Moor & $18.5 \%$ & 0.253 & 2.684 & 0.493 & 14.608 \\
\hline & Tamil & $8.0 \%$ & 0.166 & 0.300 & 0.054 & 1.651 \\
\hline & Sinhalese & $18.0 \%$ & - & - & - & - \\
\hline \multirow[t]{5}{*}{ Highest level of education } & None or Primary & $14.8 \%$ & 0.511 & 0.651 & 0.181 & 2.341 \\
\hline & Secondary & $19.8 \%$ & 0.778 & 0.884 & 0.375 & 2.085 \\
\hline & GCE OL passed & $17.3 \%$ & 0.868 & 1.075 & 0.459 & 2.519 \\
\hline & GCE AL passed & $16.7 \%$ & 0.867 & 1.064 & 0.512 & 2.213 \\
\hline & Degree and above & $17.6 \%$ & - & - & - & - \\
\hline \multirow[t]{6}{*}{ Nature of employment } & Full-time public sector & $17.7 \%$ & 0.227 & 1.518 & 0.771 & 2.989 \\
\hline & Full-time private sector & $19.7 \%$ & 0.082 & 2.075 & 0.912 & 4.717 \\
\hline & $\begin{array}{l}\text { Self-employed / own } \\
\text { account employer }\end{array}$ & $15.3 \%$ & 0.394 & 1.357 & 0.672 & 2.741 \\
\hline & Unpaid family work & $28.6 \%$ & 0.091 & 2.642 & 0.856 & 8.149 \\
\hline & Retired & $11.1 \%$ & 0.759 & 1.378 & 0.178 & 10.693 \\
\hline & Unemployed & $17.4 \%$ & - & - & - & - \\
\hline \multirow[t]{5}{*}{ Monthly income (LKR) } & $<20,000$ & $23.9 \%$ & 0.013 & 3.922 & 1.340 & 11.478 \\
\hline & 20,000-39,999 & $16.2 \%$ & 0.017 & 3.131 & 1.223 & 8.017 \\
\hline & $40,000-59,999$ & $19.0 \%$ & 0.014 & 3.121 & 1.261 & 7.726 \\
\hline & $60,000-79,999$ & $18.9 \%$ & 0.014 & 3.763 & 1.306 & 10.845 \\
\hline & $80,000 \&$ above & $10.5 \%$ & - & - & - & - \\
\hline \multirow[t]{2}{*}{ Residential sector } & Rural & $15.6 \%$ & 0.585 & 0.878 & 0.549 & 1.402 \\
\hline & Urban & $18.7 \%$ & - & - & - & - \\
\hline
\end{tabular}

\footnotetext{
${ }^{a}$ As a proportion of total investigated
}

${ }^{\mathrm{b}} \mathrm{P}$ values are for the adjusted odds ratios based on the multiple logistic regression analyses. The dependent variable was defined as 1 if undiagnosed hypercholesterolaemia, and 0 if previously diagnosed hypercholesterolaemia. The odds ratio for a given variable was adjusted for all other co-variables in the table. 


\section{Discussion}

This study revealed high proportions of undiagnosed CVD risk factors in an overweight/obese subpopulation, amounting to $13.8 \%$ (95\% CI: 11.9, 15.8) for diabetes, $11.3 \%$ (95\% CI: 9.5, 13.1) for hypertension and $17.8 \%$ (95\% CI: 15.6, 19.9) for hypercholesterolaemia. Undiagnosed cases represent the unseen but clinically important burden of risk factors, with significant concurrent metabolic derangements and a long-term impact on health care use $[14,15]$. Of the three risk conditions, hypercholesterolaemia was the most undiagnosed condition, whereas hypertension was the least undiagnosed condition. This discrepancy reflects the differences in accessibility and affordability to diagnostic facilities.

According to the global burden of disease analysis, hypertension is the leading single risk factor responsible for preventable global CVD burden. Other risk factors include an unhealthy diet, high total cholesterol, air pollution, tobacco use, high body mass index, high fasting plasma glucose, impaired kidney function, and low physical activity in that order [2]. The present analysis focused on three of those important risk factors which need laboratory testing at a health facility.

In our definition of 'undiagnosed proportion', we used the total number investigated as the denominator as opposed to the total number with the respective disease. This definition is more relevant in community screening programmes since it reflects the probability that a person in the population has the disease without her or his knowledge. Such people would continue without treatment until they are detected at a later stage with irreversible complications such as CVD, stroke, or kidney disease. Thus, the proportion 'undiagnosed' based on a community sample like in this would be a useful indicator to evaluate the effectiveness of screening programmes in detecting CVD risk in the community.

This study revealed an alarmingly high prevalence of diabetes, hypertension, and hypercholesterolaemia, $28.0 \%$, 33.4\%, and $31.9 \%$, respectively. Our findings, together with other available studies, confirm that there is a rapidly rising trend in diabetes, especially in urban settings [11,16-19]. With this trend, the undiagnosed population may rise even faster. According to our findings, undiagnosed diabetes accounted for almost half of all diabetes cases, compared to one-third reported previously [11]. In contrast, undiagnosed hypertension accounted for one-third of all hypertension cases in the present study compared to half reported previously [17].

We identified some relevant determinants of undiagnosed CVD risk factors through regression analyses while addressing the confounding effects. The key determinants for undiagnosed CVD were: male sex, low or middle income, rural residence, and relatively younger age. Living in a rural area as a predictor for undiagnosed DM could be attributed to a lack of motivation or low accessibility to blood glucose testing services in a rural setting compared to the urban. The costs also play an independent role in undiagnosed diabetes, indicating less affordability. Thus, it is important to ensure that all primary health care facilities in a rural setting will have testing facilities, and people are motivated to attend such services.

Male sex as a predictor for undiagnosed hypertension indicates that men are ignorant about the screening facilities compared to women. Employed status is a major barrier that prevents men from attending these services during working hours. However, there were no differences across the type of employment in the present analysis. Our findings suggest the need to encourage and provide provisions for men in accessing services for blood pressure checking, preferably at the workplace or during nonworking hours at HLC.

Lower household income as a predictor of undiagnosed diabetes and elevated cholesterol indicates an economic reason for poor detection. Concerning cholesterol, the odds are very high even for middle-income groups compared to the highest income group. This raises concerns whether testing facilities are not readily available at the government health facilities and people cannot afford high cost for testing lipid profiles at private sector laboratories. However, it is important to provide this service in all state health facilities or subsidize it for lowincome groups.

Our analysis found that older ages were less likely to be undiagnosed compared to younger ages. This is explained by the frequent health care seeking by the elderly. The age-specific analyses show a rapid increase in obesity, diabetes, and hypertension during the $4^{\text {th }}$ decade of life [20]. Therefore, screening programmes should target lower ages, especially those around 40 years.

A mere increase in coverage of screening at primary health care facilities does not guarantee that it would capture many of those with CVD conditions in the population. If the attendees are of lower risk, then there will not be many positives despite a high screening coverage. Therefore, screening programmes should adopt a high-risk screening strategy, where relevant, focusing on the determinants of undiagnosed CVD risk factors. Tailoring screening programmes according to contexts such as risk level to reach those most in need has been recommended in a review on effectiveness and uptake of screening programmes for coronary heart disease and diabetes globally [21]. In Sri Lanka, there is a promising initiative by the state health services to enhance primary health care services in the recent past. The cluster care system with defined catchment areas for each PHC aims for high coverage of screening and detection of CVD risk in the population [22]. We suggest targeting populations based on the determinants highlighted by this study to enhance its efficiency. Thereby, detection rates of diabetes 
mellitus, hypertension, dyslipidaemia can be improved in cluster catchment areas. Such an approach would contribute to reducing premature deaths due to cardiovascular diseases in the country.

The present study has a few limitations. The sample of the study is representative, however, the representativeness was affected somewhat due to 2 reasons: selection of houses located together within the cluster; and less availability of males than females during home visits. However, males were approached at their workplace or home during non-working days. Our study population is different from the general population since we selected individuals with a BMI over $25 \mathrm{~kg} / \mathrm{m}^{2}$. This resulted in a sex imbalance with a higher proportion of females in the sample, which was addressed in the regression analysis by adjusting for sex and other covariates. The study did not include access to health care services as a key determinant in the analyses.

\section{Conclusions}

The present study revealed high proportions of undiagnosed diabetes, hypertension, and hypercholesterolaemia in an overweight/obese population. Male sex, low or middle income, rural residence, and relatively younger age were the key determinants for undiagnosed CVD risk factors. The CVD screening programmes should be tailored to target populations based on these determinants and provide basic diagnostic facilities in all health centres. The 'proportion undiagnosed' in the population may be a useful indicator to evaluate the effectiveness.

\section{Acknowledgements}

This study was funded by the National Research Council Sri Lanka (NRC-15-145), with additional support from the University Research Grants (AP/3/2/2017/SG/08), Global Health Research Unit on Diabetes and Cardiovascular Disease in South Asians, and laboratory support from the Medical Research Institute (MRI 11-2018). The authors wish to acknowledge the Ministry of Health and health officials of the Western Province for granting permission and facilitating field work of this study. Authors also appreciate the administrative support by Ms. Krishni Fernando and Mr. Nimal Rathnasiri.

\section{References}

1. Roth GA, Johnson C, Abajobir A, Abd-Allah F, Abera SF, Abyu G, et al. Global, Regional, and National Burden of Cardiovascular Diseases for 10 Causes, 1990 to 2015. J Am Coll Cardiol 2017; 70(1): 1-25.

2. Thomas H, Diamond J, Vieco A, Chaudhuri S, Shinnar E, Cromer S, et al. Global Atlas of Cardiovascular Disease 2000-2016: The Path to Prevention and Control. Glob Heart 2018; 13(3): 143-63.
3. Minsitry of Health Nutrition and Indigenous Medicine. Annual Health Statistics, 2017. Colombo, 2019.

4. United Nations. Sustainable Development Goals. 2015. https://sustainabledevelopment.un.org/?menu=1300 (accessed 2 Apr 2020).

5. Yusuf S, Hawken S, Ounpuu S, Dans T, Avezum A, Lanas F, et al. Effect of potentially modifiable risk factors associated with myocardial infarction in 52 countries (the INTERHEART study): case-control study. Lancet 2004; 364: 937-52. doi:10.1016/S0140-6736(04) 17018-9.

6. World Health Organization. Cardiovascular diseases. Fact sheet. 2017. https://www.who.int/news-room/fact-sheets/ detail/cardiovascular-diseases-(cvds) (accessed 2 Apr 2020).

7. Mallawaarachchi DSV, Wickremasinghe SC, Somatunga LC, Siriwardena VT, Gunawardena NS. Healthy Lifestyle Centres: a service for screening noncommunicable diseases through primary health-care institutions in Sri Lanka. WHO South-East Asia J Public Heal 2016; 5(2): 89-95.

8. Ministry of Health Nutrition and Indigenous Medicine. Non-communicable disease risk factor survey, Sri Lanka. Colombo, 2015.

9. Ministry of Health Nutrition and Indigenous Medicine, Department of Census and Statistics, World Health Organization, Global Fund to Fight AIDS, Tuberculosis and Malaria KMS. Service Availability and Readiness Assessment Sri Lanka 2017. Colombo, 2018.

10. Selvin E, Wang D, Lee AK, Coresh J, Bergenstal RM. Identifying Trends in Undiagnosed Diabetes in U.S. Adults. Ann Intern Med 2018; 168(12): 900-1.

11. Katulanda P, Constantine GR, Mahesh JG, Sheriff R, Seneviratne RDA, Wijeratne S, et al. Prevalence and projections of diabetes and pre-diabetes in adults in Sri Lanka - Sri Lanka Diabetes, Cardiovascular Study (SLDCS). Diabet Med 2008. doi:10.1111/j.1464-5491.2008.02523.x.

12. Senarath U, Katulanda P, Fernando DN, Kalupahana NS, Partheepan K, Jayawardena R, et al. mHealth nutrition and lifestyle intervention (mHENAL) to reduce cardiovascular disease risk in a middle-aged, overweight and obese population in Sri Lanka: Study protocol for a randomized controlled trial. Contemp Clin Trials Commun 2019; 16. doi:10.1016/j.conctc.2019.100453.

13. American Diabetes Association. Classification and diagnosis of diabetes: Standards of medical care in diabetes-2019. Diabetes Care 2019; 42(Suppl.1 - January): S13-S28.

14. Young TK, Mustard CA. Undiagnosed diabetes: Does it matter? 2001; 164(1).

15. Skeldon SC, Detsky AS, Goldenberg SL, Law MR. Erectile dysfunction and undiagnosed diabetes, hypertension, and hypercholesterolemia. Ann Fam Med 2015; 13(4): 331-5.

16. De Silva AP, De Silva SHP, Haniffa R, Liyanage IK, Jayasinghe KSA, Katulanda P, et al. A survey on socioeconomic determinants of diabetes mellitus management in a lower middle income setting. Int $J$ Equity Health 2016; 15(1). doi:10.1186/s12939-016-0363-3.

17. Katulanda P, Ranasinghe P, Jayawardena R, Constantine GR, Rezvi Sheriff MH, Matthews DR. The prevalence, 
predictors and associations of hypertension in Sri Lanka: A cross-sectional population based national survey. Clin Exp Hypertens 2014. doi:10.3109/10641963.2013.863321.

18. Katulanda P, Dissanayake HA, De Silva SDN, Katulanda GW, Liyanage IK, Constantine GR, et al. Prevalence, patterns, and associations of dyslipidemia among Sri Lankan adults - Sri Lanka Diabetes and Cardiovascular Study in 2005-2006. J Clin Lipidol 2018; 12(2). doi:10.1016/j.jacl. 2018.01.006.

19. Somasundaram NP, Ranathunga I, Gunawardana K, Ediriweera DS. High prevalence of diabetes mellitus in Sri Lankan urban population - data from Colombo urban study. Sri Lanka J Diabetes Endocrinol Metab 2019; 9(2): 8.
20. Gamlath L, Nandasena S, Hennadige Padmal De Silva S, Linhart C, Ngo A, Morrell S, et al. Differentials in Cardiovascular Risk Factors and Diabetes by Socioeconomic Status and Sex in Kalutara, Sri Lanka. Asia-Pacific J Public Heal 2017; 29(5): 401-10.

21. Holland C, Cooper Y, Shaw R, Pattison H, Cooke R. Effectiveness and uptake of screening programmes for coronary heart disease and diabetes: a realist review of design components used in interventions. BMJ Open 2013; 3(11). doi:10.1136/bmjopen-2013-003428.

22. Ministry of Health Care and Nutrition Sri Lanka. Piloting a Primary Health Care Reform in Sri Lanka. Colombo, 2019. 